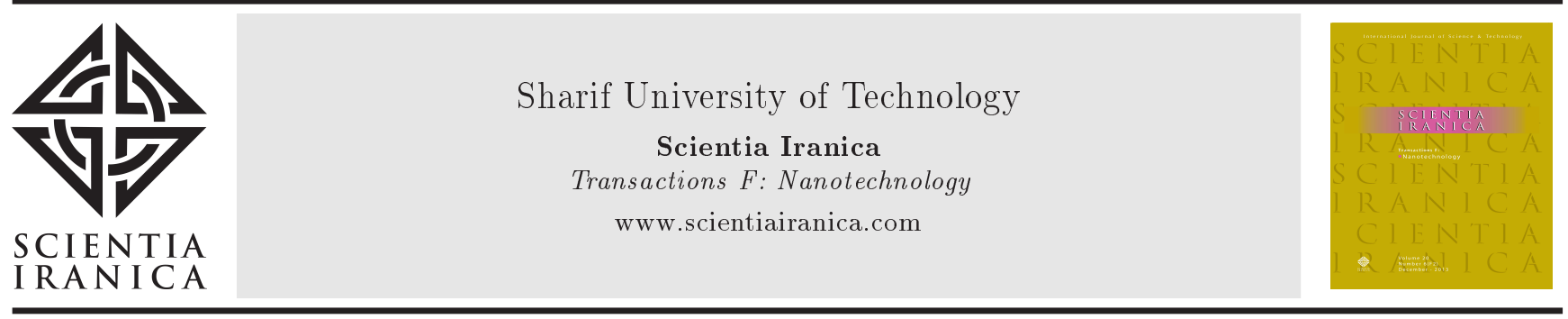

Research Note

\title{
First-principles study of strain-induced charge polarization in a molybdenum disulfide monolayer
}

\author{
N. Salami ${ }^{\mathrm{a}, *}$ and A.A. Shokri ${ }^{\mathrm{b}}$ \\ a. Department of Physics, Yasooj Branch, Islamic Azad University, Yasooj, Iran. \\ b. Department of Physics, Tehran Payame-Noor University, Tehran, P.O. Box 19395-3697, Iran. \\ Received 7 June 2016; received in revised form 24 September 2016; accepted 8 April 2017
}

\author{
KEYWORDS \\ Molybdenum \\ disulfide; \\ Charge polarization; \\ Piezoelectric; \\ Electrostatic \\ potential.
}

\begin{abstract}
In the presence of elastic planar strain distributions, electronic properties of molybdenum disulfide $\left(\mathrm{MoS}_{2}\right)$ monolayer are investigated within Density Functional Theory (DFT) calculations as implemented in SIESTA package. Three types of planar strain are considered with some different intensity values, and uniaxial strain along the armchair and zigzag directions as well as biaxial strain. We present a systematic study of the strained $\mathrm{MoS}_{2}$ monolayer by focusing on the calculation of Total Density Of State (TDOS), Partial Density Of State (PDOS), electron charge density, and electrostatic potential using post processing tools. In most cases, the states due to Mo atoms have dominant association in the TDOS close to the Fermi level of $\mathrm{MoS}_{2}$ monolayer under strain. As a consequence of the strain, $\mathrm{S}$ atom takes electron from Mo atom and becomes negatively charged. In addition, the tensile and compressive strains introduce the charge polarization in two opposite directions per three types of strain for both sheets, which is in line with the experimental study. As another important result, the strain-induced charge polarization is proportional to the intensity value of strain.
\end{abstract}

(C) 2017 Sharif University of Technology. All rights reserved.

\section{Introduction}

$\mathrm{MoS}_{2}$ single-layer, as one of transition metal dichalcogenide (TMD) monolayers (novel two dimensional honeycomb semiconductors), is composed of three layers with hexagonal symmetry, in which each Mo atom is covalently bonded with equal distances with three $\mathrm{S}$ atoms in up-layer and three $\mathrm{S}$ atoms in down-layer. It has been focused on due to remarkable properties, including sizable direct band gap [1], tunable electronic properties, high coherence length [2], easy fabrication [3-5], and high surface to volume ratio [6,7]. Also, It shows a high on/off current ratio and mobility when a hafnium oxide (high $\mathrm{k}$ dielectric) is used

*. Corresponding author. Tel.: +98 7433310493 E-mail addresses: salami@iauyasooj.ac.ir (N. Salami); aashokri@pnu.ac.ir (A.A. Shokri) as a gate dielectric [8], allowing applications in the next-generation nano-devices. In addition, defect-free $\mathrm{MoS}_{2}$ monolayer and $\mathrm{MoS}_{2}$ monolayer in the presence of single S-vacancy as well as single Mo-vacancy do not exhibit any magnetic properties [9]. However, magnetism is found in the $\mathrm{MoS}_{2}$ monolayer when the monolayer is doped by some transition metal atoms [10] and nonmagnetic elements [11], in which the induced local magnetism can be controlled via geometrical distortions [12].

The electronic properties of $\mathrm{MoS}_{2}$ monolayer can be manipulated by means of applying strain [13-18] and external electric and magnetic fields [19]. For example, the biaxial planar tensile strain induces direct to indirect band gap transition in $\mathrm{MoS}_{2}$ monolayer $[13,15]$. Also, it exhibits semiconductor-metal transition upon the biaxial planar tensile strain about $11 \%[13,14]$. Moreover, the biaxial and uniaxial tensile and compres- 
sive strains modulate longitudinal and transverse effective masses of both electron and hole [14,18]. On the other hand, both experimental and theoretical studies have reported piezoelectricity in two-dimensional $\mathrm{MoS}_{2}$ monolayer [20,21]. The observed piezoelectricity causes $\mathrm{MoS}_{2}$ monolayer to be a potential candidate for piezotronics. In order to provide deep understanding of strain-induced charge polarization as well as electrostatic potential, a systematic study is presented on the electronic properties of the strained $\mathrm{MoS}_{2}$ singlelayer [22-26].

In the earlier work, we calculated the tunable electronic and magnetic properties of an $\mathrm{MoS}_{2}$ monolayer with vacancies under elastic planar strain at the level of Density Functional Theory (DFT) [9]. Here, we provide theoretical observations of induced charge polarization as well as the electrostatic potential due to the elastic mechanical deformations in the $2 \mathrm{D} \mathrm{MoS}_{2}$ monolayer in details. All calculations are performed using DFT implemented in SIESTA package based on norm-conserving pseudopotentials to describe the electron-ion interaction and numerical atomic orbital basis set for the expansion of the single-particle KohnSham wave functions.

The outline of this paper is as follows. Technical details of DFT calculations for the $\mathrm{MoS}_{2}$ monolayer system are briefly provided in Section 2. Also, various configurations are introduced. In Section 3, the influence of the elastic planar strain on the electronic properties is investigated. The last section of the paper is allocated to a brief discussion and conclusion of the findings.

\section{Computational details}

The type and intensity dependence of strain on the electronic properties of an $\mathrm{MoS}_{2}$ monolayer is theoretically investigated by focusing on the piezoelectric charge polarization. For this reason, the structural relaxation and electronic structure calculation are performed based on the DFT implemented in the SIESTA package $[27,28]$, which uses the numerical atomic orbitals as the basis set and Troullier-Martin type normconserving pseudopotentials [29]. Also, Perdew-BurkeErnzerhof (PBE) functional form of Generalized Gradient Approximation (GGA) for the exchange-correlation approximation, which generates excellent results for TMD materials [13], has been used. Mo and S atoms are described by $5 \mathrm{~s}^{1} 4 \mathrm{~d}^{5}$ and $3 \mathrm{~s}^{2} 3 \mathrm{p}^{4}$ valence electrons plus the corresponding pseudo-potential ion charges, respectively.

We aim to study the effect of various tensile and compressive planar strain distributions on electronic properties of $\mathrm{MoS}_{2}$ single-layer. For this reason, we have considered three types of strain distribution for the system, namely, uniaxial (asymmetrical) strain distribution along the armchair direction as shown in the right panel of Figure 1(a), uniaxial (asymmetrical) strain distribution along the zigzag direction as shown in the right panel of Figure 1(b), and the symmetrical (biaxial) strain distribution as shown in the right panel of Figure 1(c). Also, the directions of three types of strain have been depicted from the viewpoint of charge density in Figure 1. The tensile and compressive planar strains, $\varepsilon \%$, along the special direction can be modeled by increasing and decreasing the lattice constant from $a$ to $a(1+\varepsilon)$ and $a(1-\varepsilon)$, respectively. After the SIESTA calculations, the results are post-processed using the pdosxml, denchar, and plrho utilities of SIESTA to calculate the Partial Density Of States (PDOS), the charge density distribution, and the electrostatic potential, respectively.

\section{Results and discussions}

In order to investigate the electronic properties of the $\mathrm{MoS}_{2}$ monolayer under different strain distributions, at first, we have represented the Total Density Of States (TDOS) of a pristine $\mathrm{MoS}_{2}$ layer in Figure 2(a). The calculations have been done on the rectangular unit cell of $\mathrm{MoS}_{2}$ single-layer with the lattice constant $5.55 \times 3.20 \times 20.00$ in unit of angstrom, which contains six atoms, while the rectangular supercell of $\mathrm{MoS}_{2}$ single-layer has been used with the lattice constant $5.55 \times 6.40 \times 20.00$ in unit of angstrom to plot the electrostatic potential. The spacing distance along $z$ direction is considered to be $20.00 \AA$ to avoid mirror interactions between the single layers. Double $\zeta$-pluspolarization basis set, plane wave cutoff of $190 \mathrm{Ry}$, and $9 \times 15 \times 1$ Monkhorst-Pack $k$-point sampling of the Brillouin zone are determined by the total energy convergence tests within $0.01 \mathrm{meV}$. However, the grid is increased to $24 \times 42 \times 1 k$-points for density-ofstate calculations. Besides these, maximum absolute forces over atoms are better than $0.004 \mathrm{eV} / \AA$ in all cases. Also, all geometry optimizations and electronic structure calculations are performed corresponding to the above considerations. The lattice constant and the band gap of the optimized pristine $\mathrm{MoS}_{2}$ monolayer are obtained $3.20 \AA$ and $1.51 \mathrm{eV}$, respectively, which are in agreement with the reported theoretical results [30]. In all calculations, the curves have been plotted with the fixed values of $\varepsilon= \pm 2 \%, \pm 5 \%$, and $\pm 12 \%$ when the strain is applied. The mechanical deformations with the considered strength values are at the elastic mechanical deformation range for the $\mathrm{MoS}_{2}$ singlelayer [13].

The TDOS of an $\mathrm{MoS}_{2}$ single-layer and its Projected Density Of States (PDOS) on the Mo and S atoms have been plotted at various intensity values of strain in Figure 1(a)-(c). It is seen that the $\mathrm{MoS}_{2}$ single-layer nearly exhibits the same electronic 

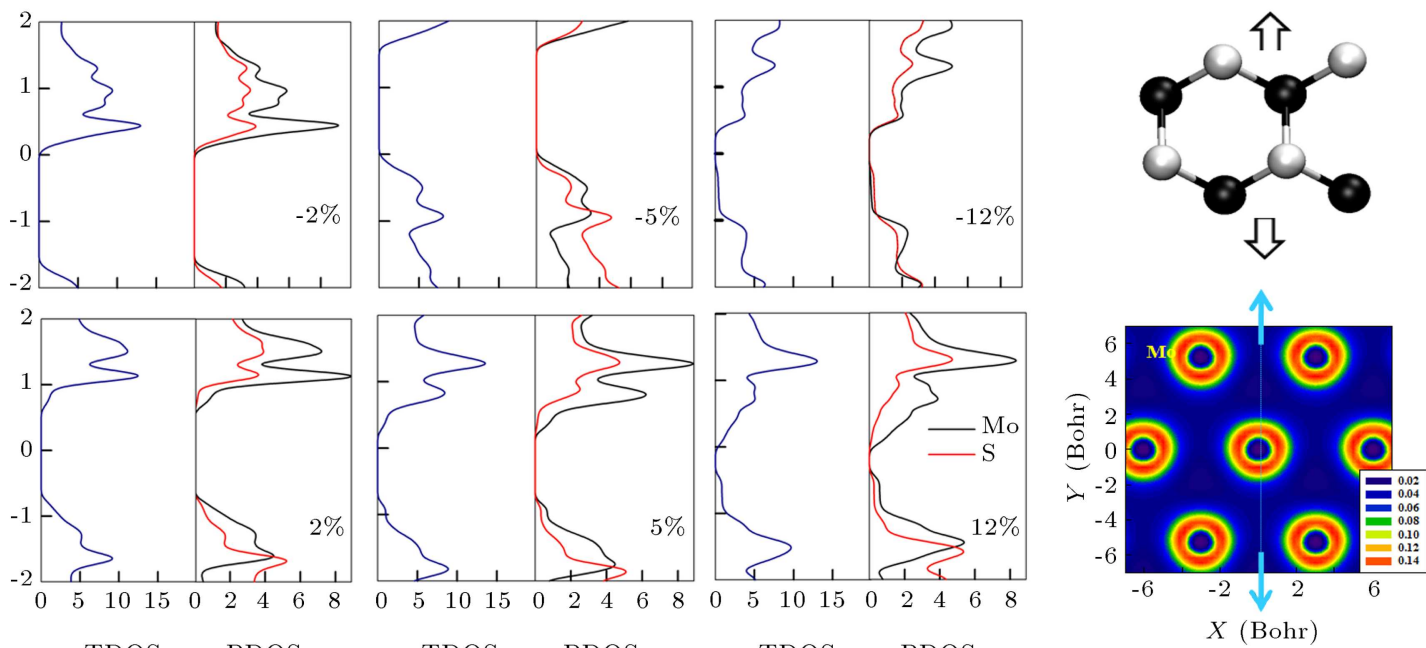

(a) Strain along armchair
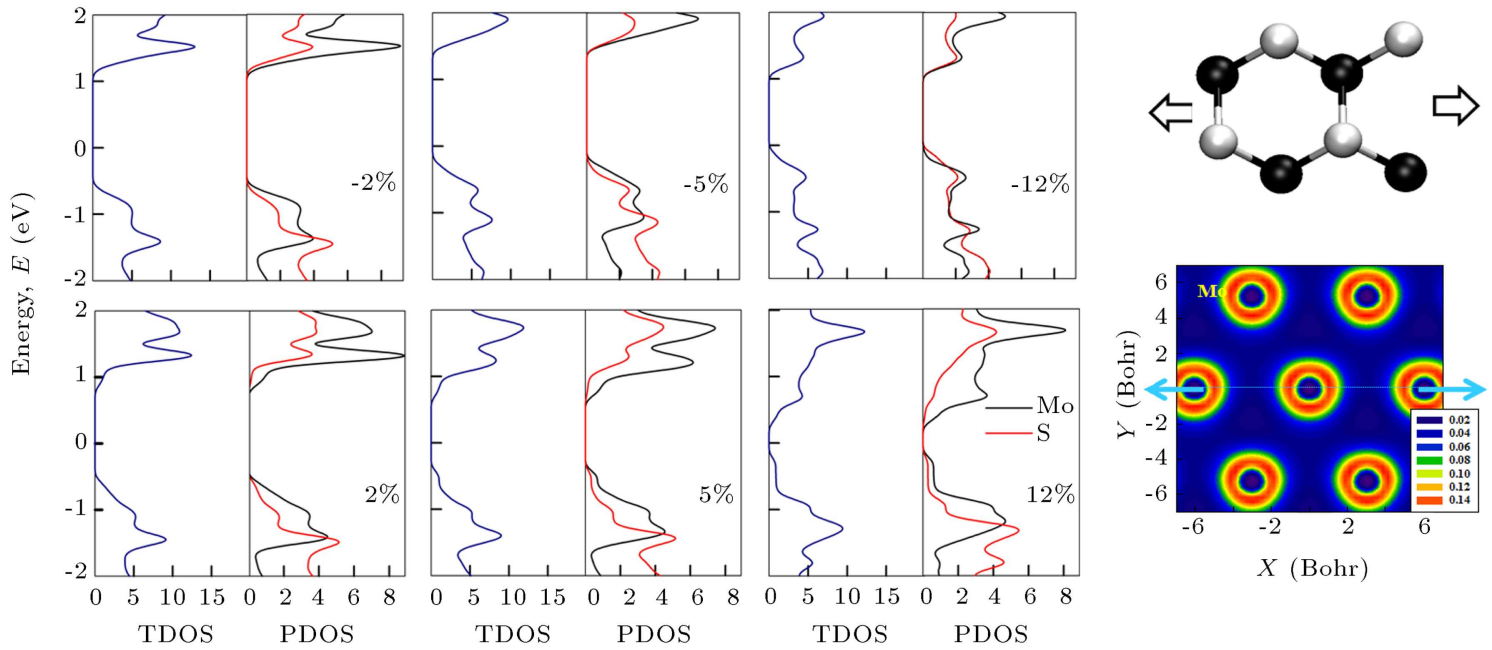

(b) Strain along zigzag
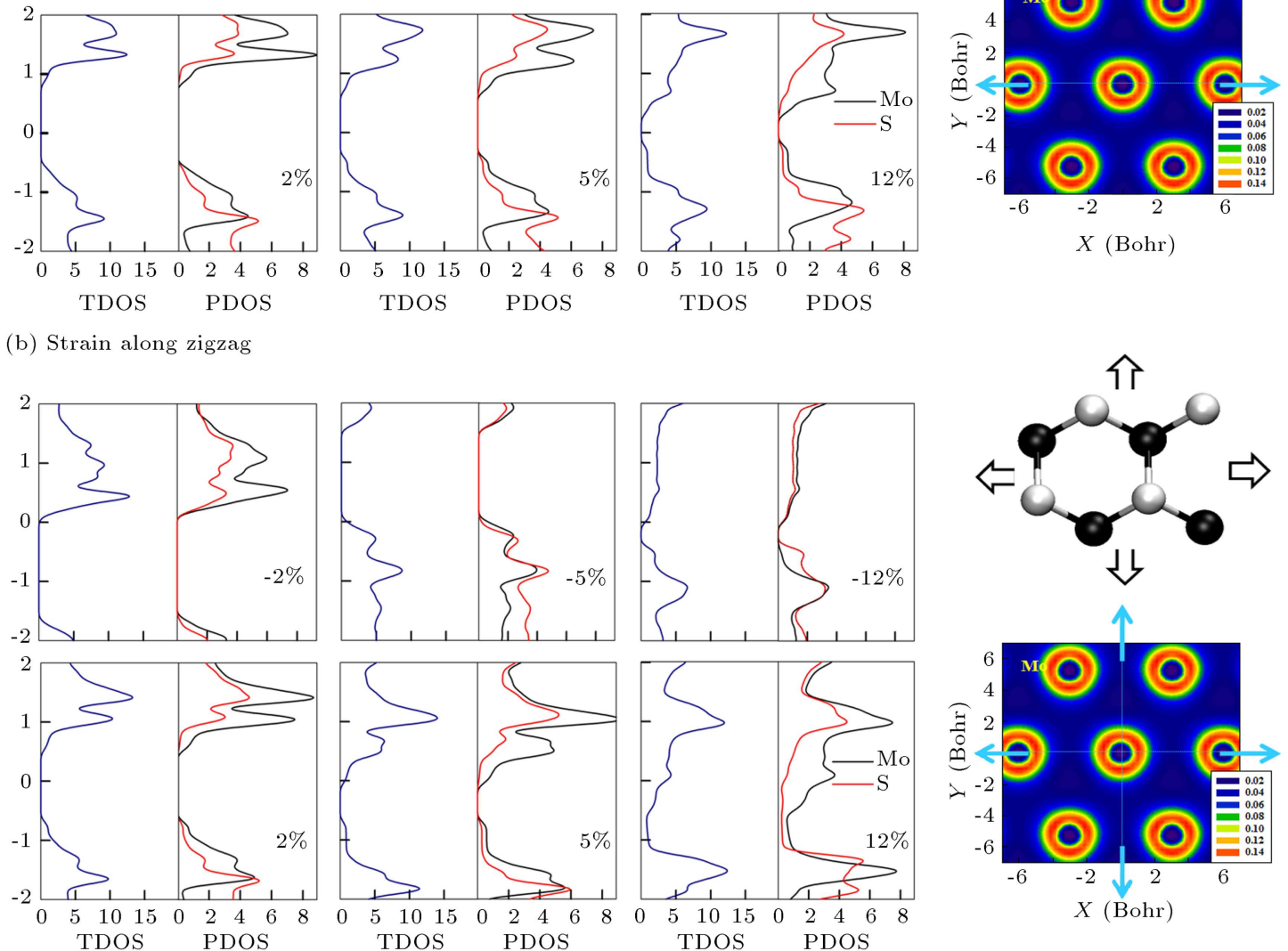

(c) Strain along biaxial

Figure 1. The dependence of the total density of states and the partial density of states on the $\mathrm{S}$ and Mo atoms as a function of energy for $\mathrm{MoS}_{2}$ monolayer under (a) the uniaxial strain along the armchair, (b) the uniaxial strain along the zigzag direction, and (c) the biaxial strain. The schematic top view of a supercell including 12 atoms and the electron charge density of Mo-Mo layer are shown in the right panel of the figure. 


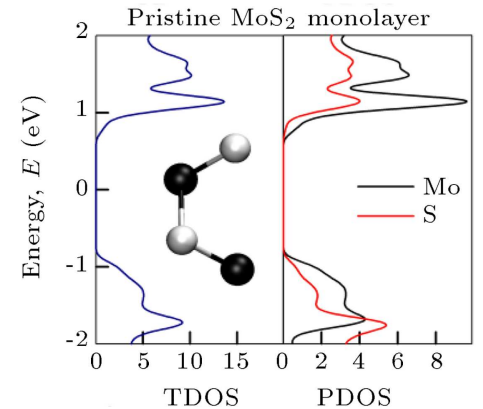

(a)

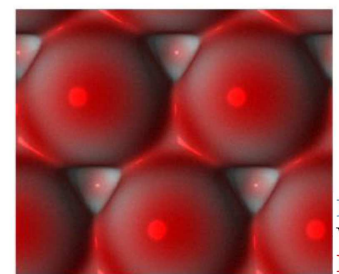

(d)

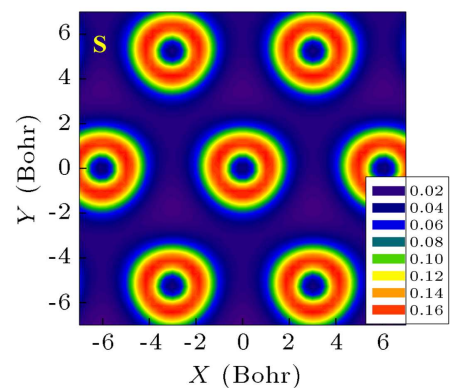

(b)

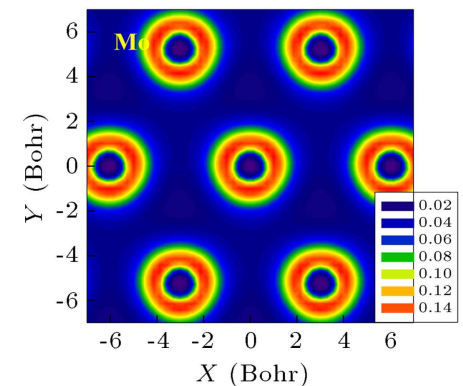

(c)

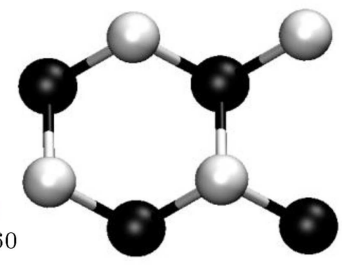

(f)

Figure 2. (a) The dependence of the total density of states as well as the partial density of states as a function of energy. The inset figure shows the hexagonal unit cell of the structure. (b) The contour plots of electron charge density of S-S sheet and (c) Mo-Mo sheet. (d) The electrostatic potential maps per isosurface of the electron density in three dimensions with the isovalues of 0.02 electrons $/$ bohr $^{3}$ and (e) 0.1 electrons $/$ bohr $^{3}$ of the pristine molybdenum disulfide monolayer. (f) Schematic representation of a rectangular supercell including 12 atoms from top view. The units of the electron density and the potential are electrons $/ \mathrm{bohr}^{3}$ and $\mathrm{Ry}$, respectively.

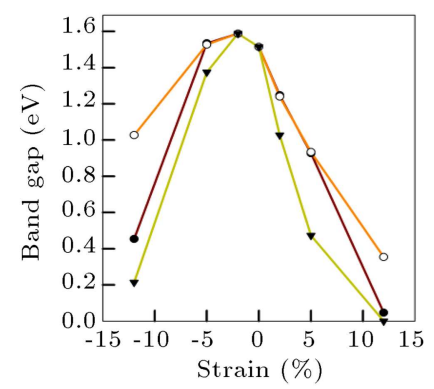

(a)

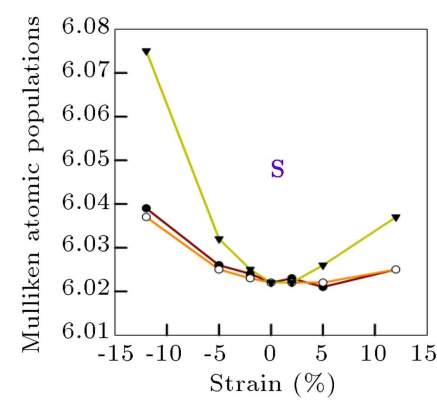

(b)

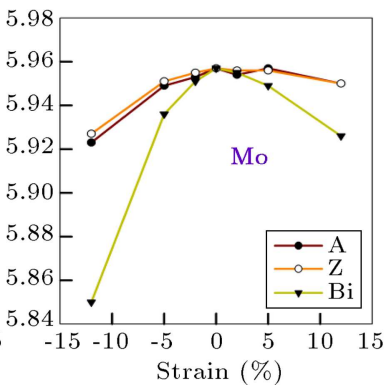

(c)

Figure 3. (a) The dependence of the band gap. Mulliken atomic population of (b) S atom and (c) Mo atom as a function of three types of stain upon the $\mathrm{MoS}_{2}$ monolayer.

properties under both of the uniaxial strains, while it is affected more due to the biaxial strain. As it is observed, the band gap of $\mathrm{MoS}_{2}$ single-layer is highly sensitive to the type and strength of strain distributions. This can be found quantitatively in Figure 3(a), which is explained in the next paragraph. Besides these, the PDOSs generally indicate that the states due to the Mo atoms have dominant association of TDOSs near the Fermi level in most cases. Also, the introduced metallic states upon the tensile biaxial strain mostly originate from the Mo atoms. The semiconductor-metal transition due to the biaxial strain is in agreement with the work of Ghorbani-Asl et al. [13].

Figure 3(a) shows the band gap evolution of $\mathrm{MoS}_{2}$ monolayer versus the intensity value of strain. In particular, one can observe that the band gap decreases upon the tensile symmetrical strain; ultimately, the $\mathrm{MoS}_{2}$ single-layer exhibits the metallic behavior under the tensile biaxial strain $\varepsilon=12 \%$, whereas the compressive biaxial strain of $\varepsilon=-2 \%$ at first causes the band gap to increase; but, the increase in the compressive strain causes the band gap to decrease, of which the trend is in agreement with the theoretically reported works [16,17]. Figure 3(b) and (c) shows the charge evolution of $\mathrm{S}$ and Mo atoms upon three types of strain, which is estimated using the Mulliken population analysis. As a consequence of the strain, $\mathrm{S}$ atom takes electron from Mo atom and becomes negatively charged. At the same time, the compressive strain induces larger partial charge changes than the tensile strain does. Also, the biaxial 

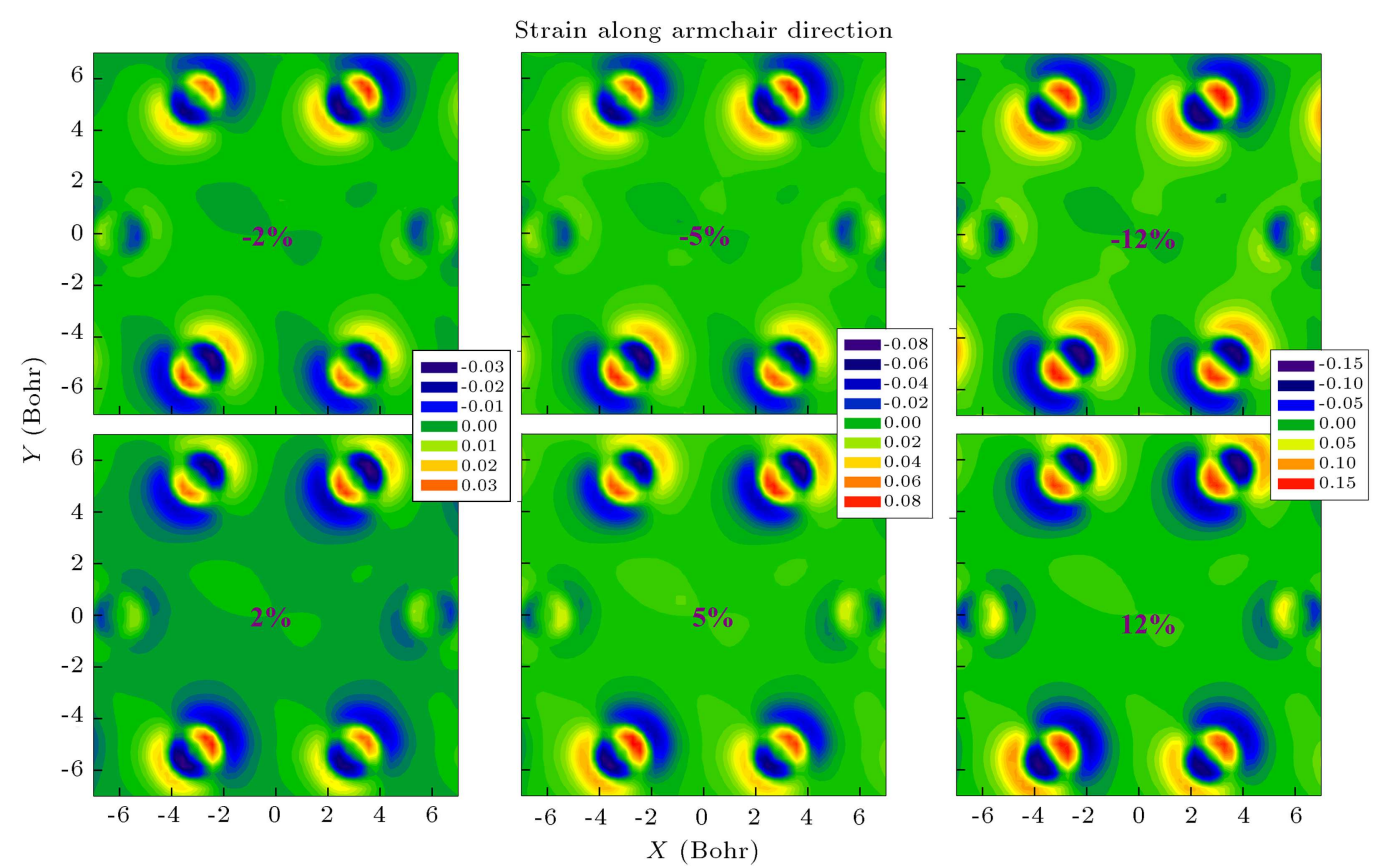

(a) Mo-Mo
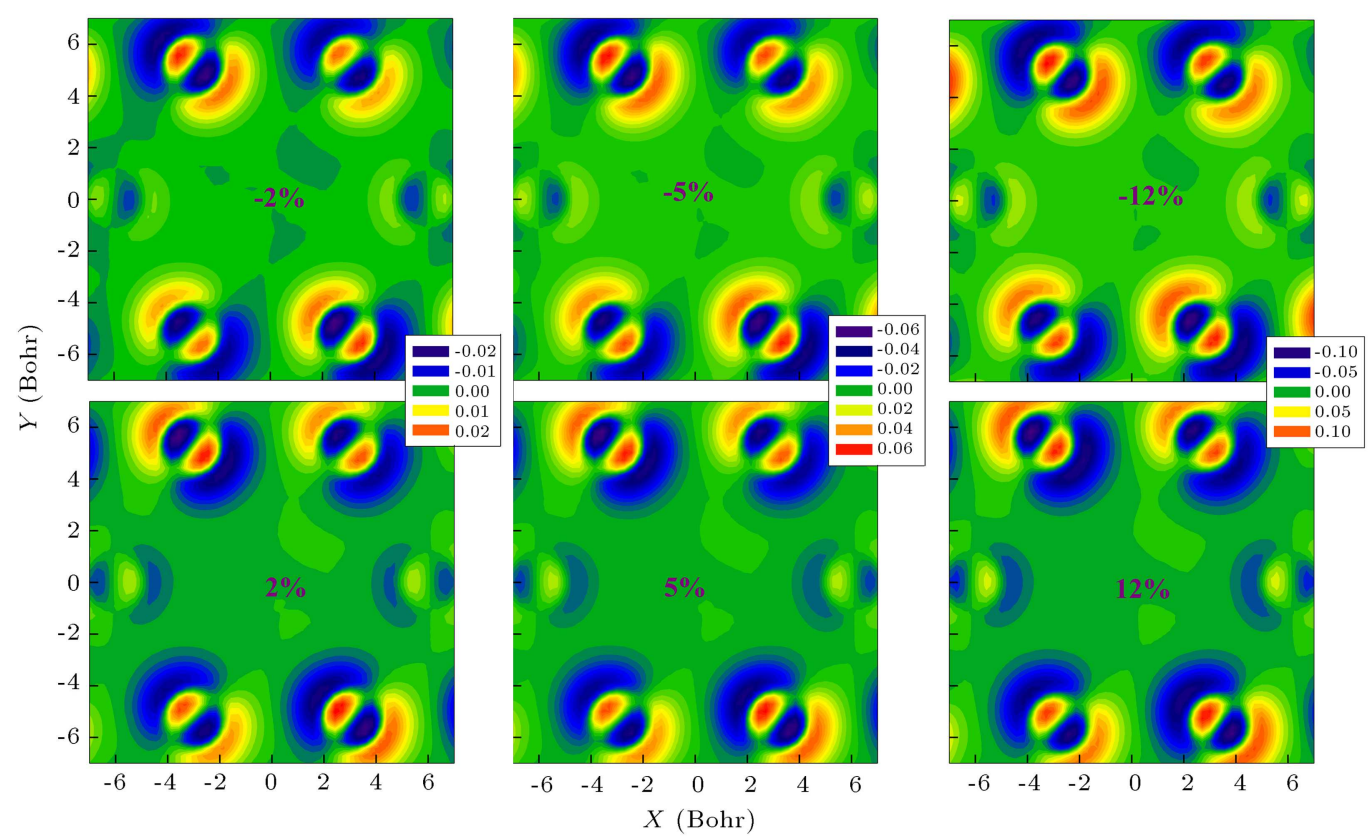

(b) S-S

Figure 4. The contour curves for electron charge density difference of (a) Mo-Mo sheet and (b) S-S sheet in MoS 2 monolayer in the presence of the strain along the armchair direction. The charge density is in the unit of electrons/bohr ${ }^{3}$.

mechanical deformation causes the atomic charge to change much more than uniaxial ones.

In order to further qualitatively elucidate the charge changes due to the applied strain, we provide the contour curves for electron charge density difference. These curves are plotted for S-S and Mo-Mo sheets of these configurations in unit of electrons/bohr ${ }^{3}$ as shown in Figures 4-6. Here, the electron charge density difference is obtained by subtracting the electron charge density of the strained $\mathrm{MoS}_{2}$ monolayer from that of pristine $\mathrm{MoS}_{2}$ monolayer. To show exact position of atoms, the contour plots of electron charge density of S-S as well as Mo-Mo sheets are plotted in Figure 2(b) and (c), respectively, in which the reference of Mo-Mo and S-S sublattices has been considered on Mo and $\mathrm{S}$ atoms, respectively. Therefore, the charge density figures as well as charge density difference figures of S-S sublattice in comparison with those of Mo-Mo sublattice have about 2 bohr displacements. In order to explore the charge polarization calculation of the 


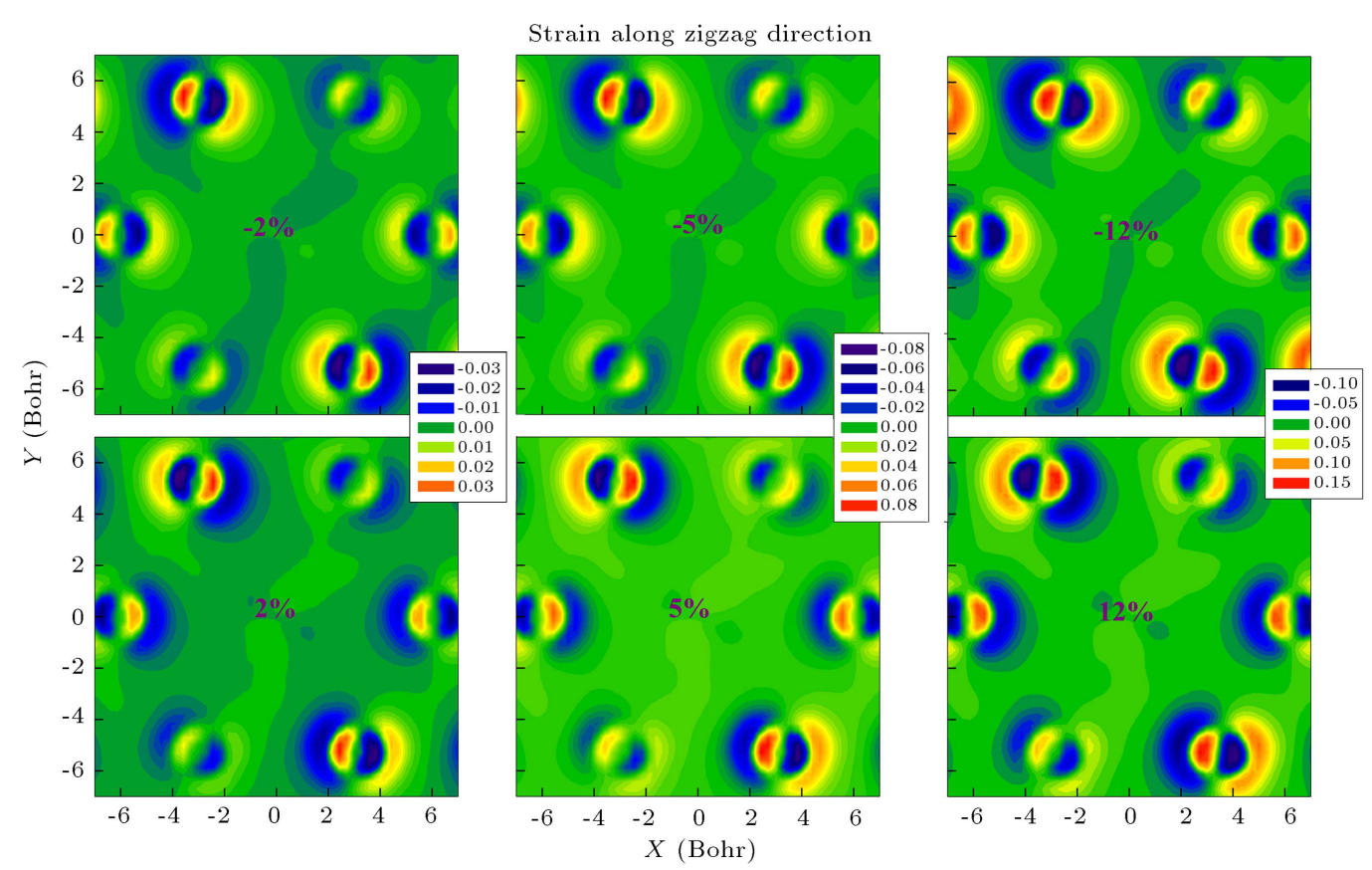

(a) Mo-Mo
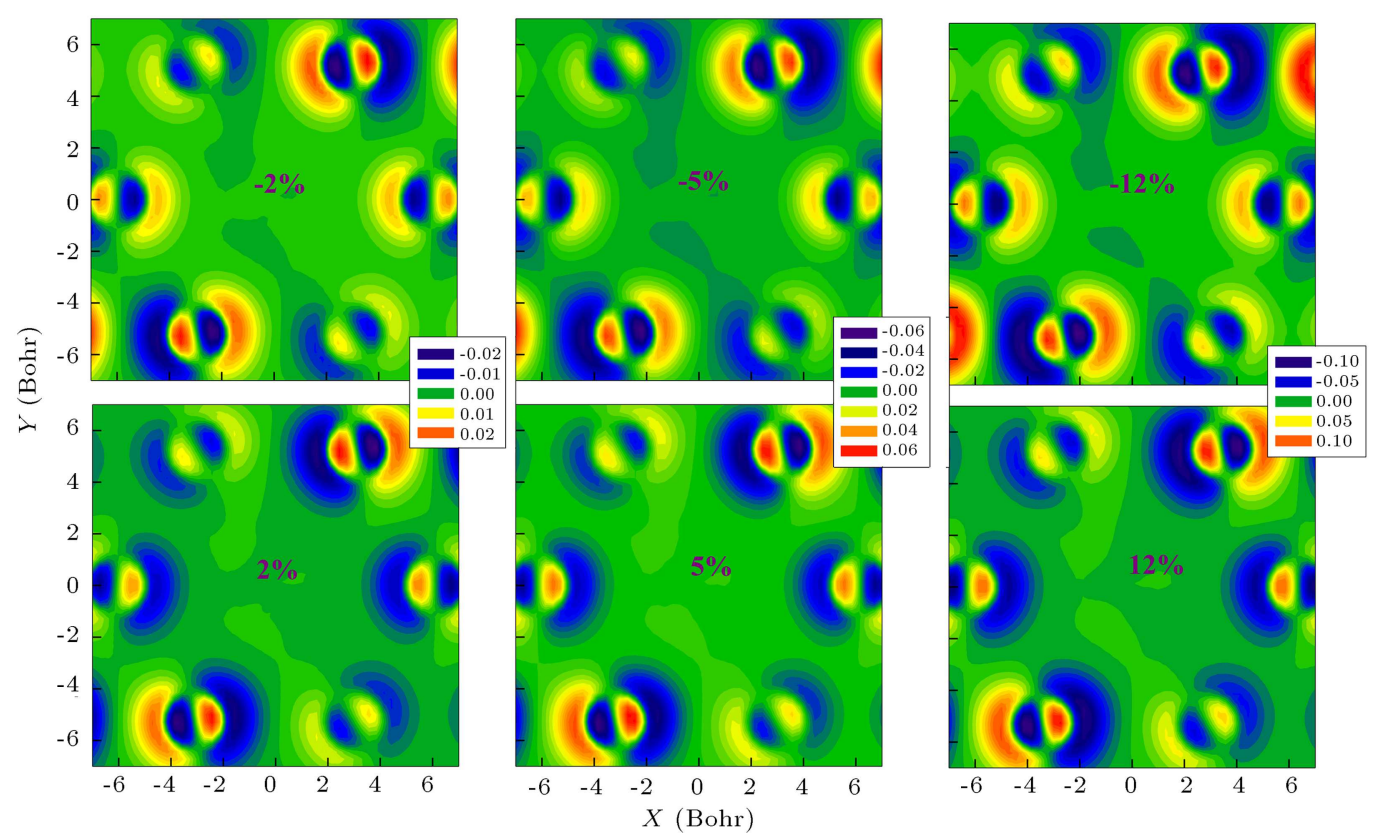

(b) S-S

Figure 5. The contour curves for electron charge density difference of (a) Mo-Mo sheet and (b) S-S sheet in MoS 2 monolayer in the presence of the strain along the zigzag direction. The charge density is in the unit of electrons/bohr ${ }^{3}$.

system, let us consider 3D lattice of $\mathrm{MoS}_{2}$ monolayer including alternating anions and cations. The first thing to point out is that there is not any Mo ion as center of inversion symmetry, which means that there is no similarity, when one sits on each Mo ion and looks at top along each Mo-S bond length and then looks at its opposite direction. Therefore, the $\mathrm{MoS}_{2}$ monolayer is polar. Polarization is defined as the dipole moment per unit area, which is measured using Sawyer-Tower circuit experimentally [31]. Hence, the change of charge polarization is computed at the edge of the $\mathrm{MoS}_{2}$ unit cell according to the figures. The figures clearly show that the strain significantly affects the electron distribution. The planar strain generally causes Mo atom to donate more charge to $\mathrm{S}$ atom, which makes the system polarize. One observes that the tensile and compressive strains induce the charge polarization in two opposite directions per three types of strains for both sheets, which is in line with the experimental study [20]. Thus, strain can asymmetrically modulate the conductance 

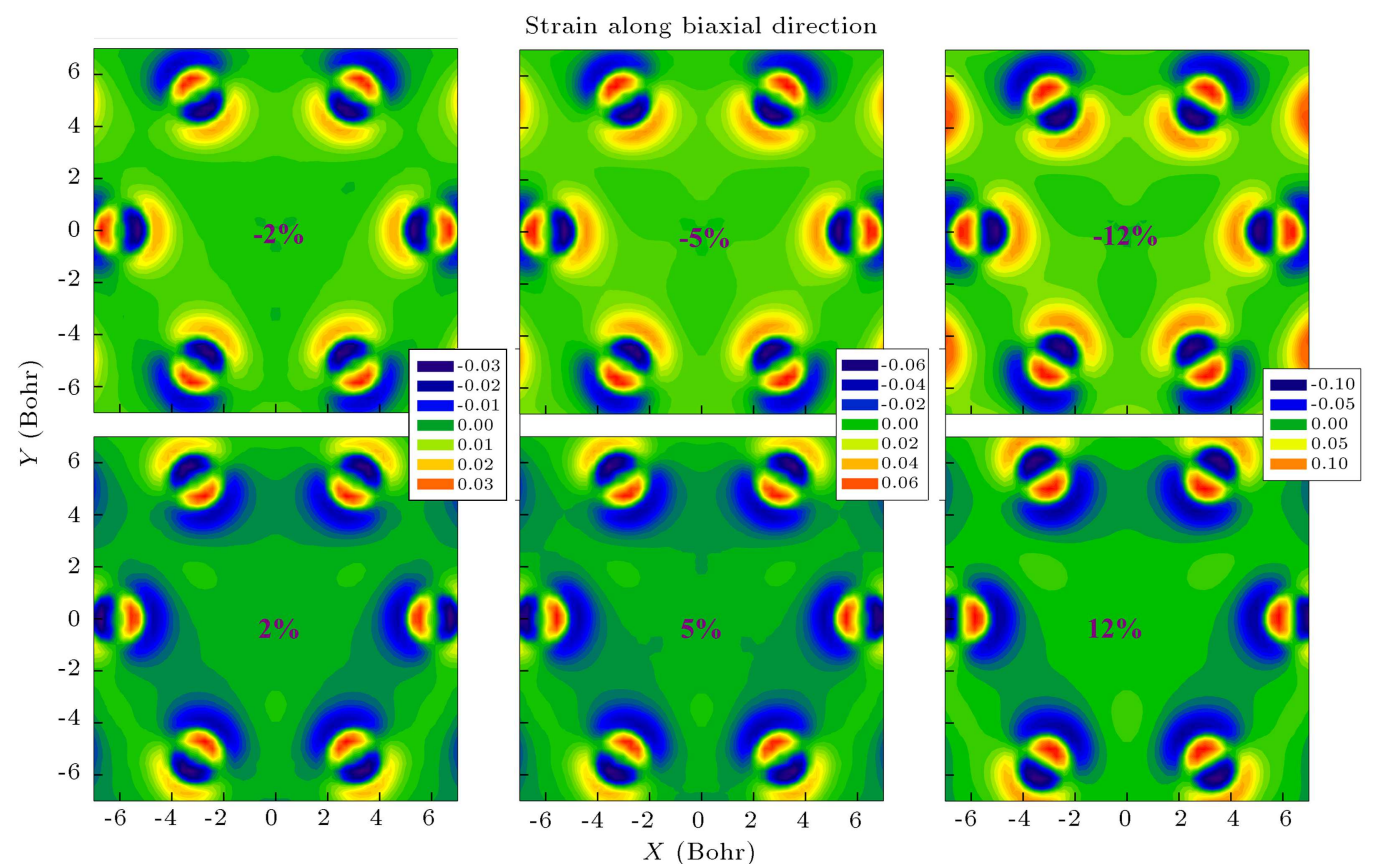

(a) Mo-Mo
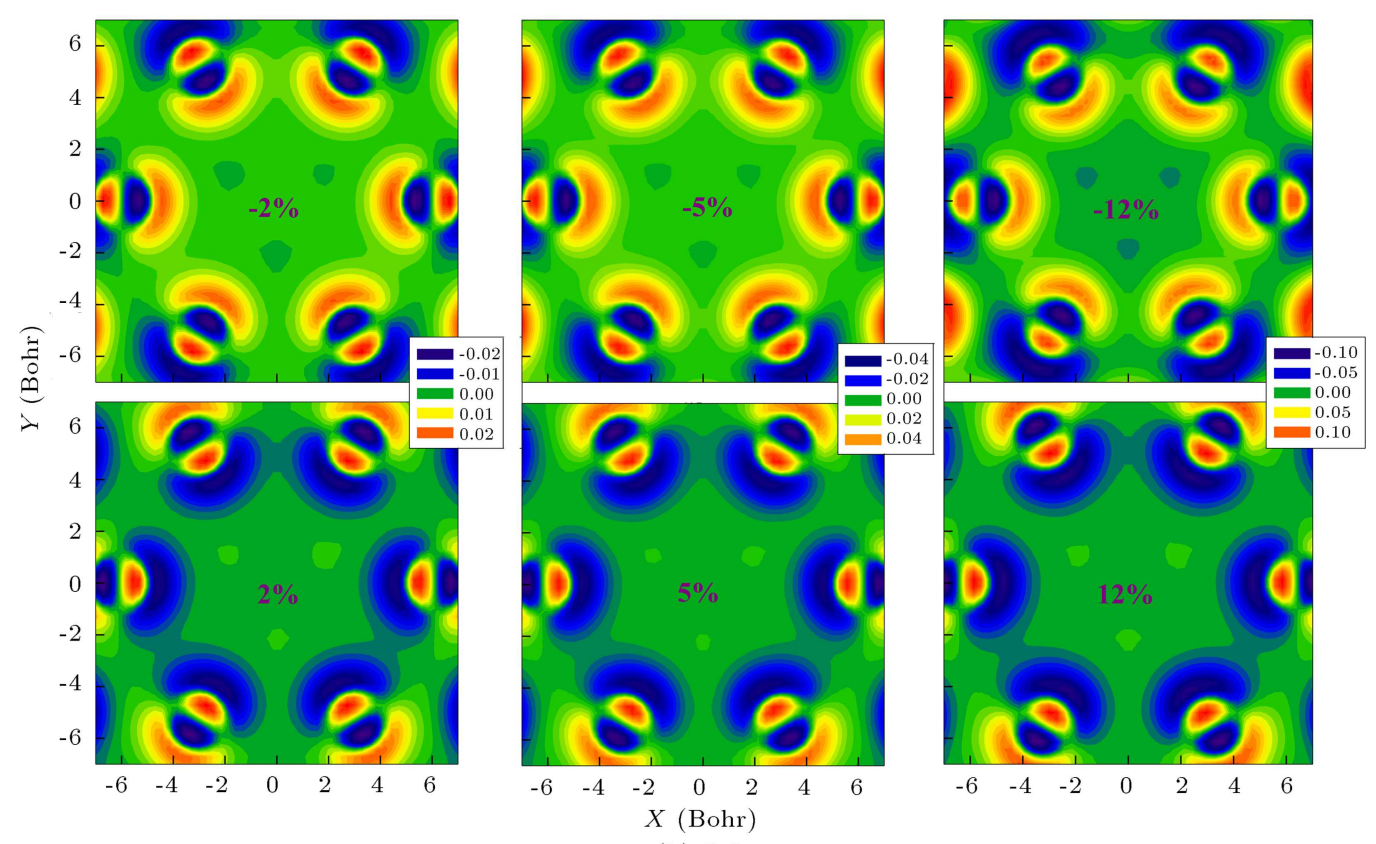

(b) S-S

Figure 6. The contour curves for electron charge density difference of (a) Mo-Mo sheet and (b) S-S sheet in MoS 2 monolayer in the presence of the biaxial strain. The charge density is in the unit of electrons $/ \mathrm{bohr}^{3}$.

of an $\mathrm{MoS}_{2}$ single-layer. As another important result, the strain-induced charge polarization is proportional to the intensity value of strain.

In order to gain insight into induced potential due to the piezoelectric charge polarization, we draw the electrostatic potential in the studied configurations using plrho code. By the way, an isosurface of the electron density is plotted in three dimensions. Then, this isosurface is colored according to the electrostatic potential of the supercell by red, white, and blue corresponding to the minimum, mean, and maximum values of the saturation range, respectively. Here, the supercell is composed of one unit cell along the armchair direction and two unit cells along the zigzag direction (see Figure 2(f)). As it is expected from the studied charge polarization, Mo atom possesses higher potential than $\mathrm{S}$ atom does. Therefore, the straininduced potential drop is created between Mo-Mo and $\mathrm{S}-\mathrm{S}$ sheets. Also, there is not any difference between the potential drop of Mo atom and S atom in up-layer and $\mathrm{S}$ 

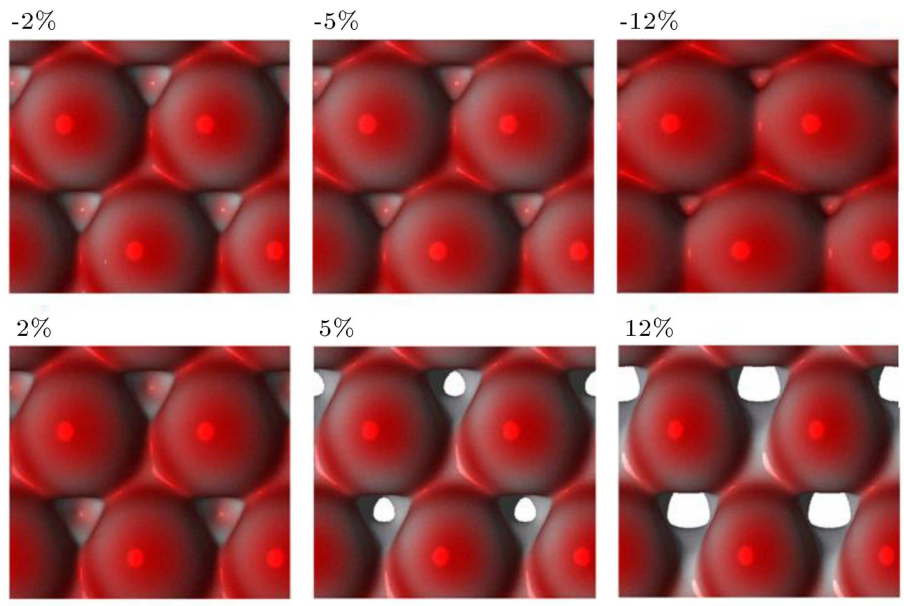

$\begin{array}{cccc} & \text { Red } & \text { White } & \text { Blue } \\ -2 \% & 0.08 & 0.18 & 3.44 \\ -5 \% & 0.08 & 0.18 & 3.46 \\ -12 \% & 0.09 & 0.20 & 3.40 \\ 2 \% & 0.08 & 0.22 & 3.45 \\ 5 \% & 0.08 & 0.18 & 3.46 \\ 12 \% & 0.08 & 0.19 & 3.47\end{array}$
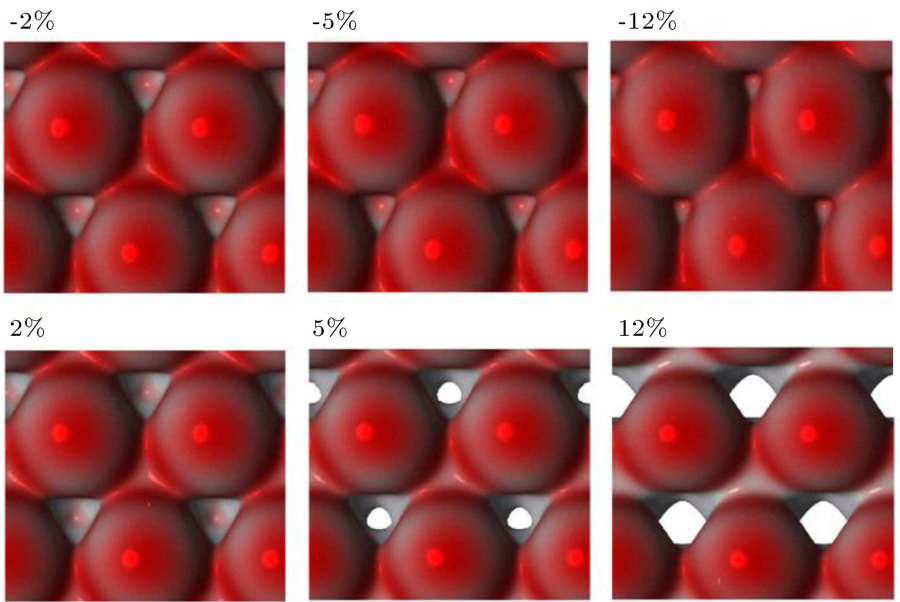

$12 \%$

$\begin{array}{cccc} & \text { Red } & \text { White } & \text { Blue } \\ -2 \% & 0.08 & 0.18 & 3.44 \\ -5 \% & 0.08 & 0.18 & 3.43 \\ -12 \% & 0.08 & 0.18 & 3.43 \\ 2 \% & 0.08 & 0.18 & 3.45 \\ 5 \% & 0.08 & 0.18 & 3.46 \\ 12 \% & 0.08 & 0.20 & 3.47\end{array}$

(b) Strain along zigzag direction
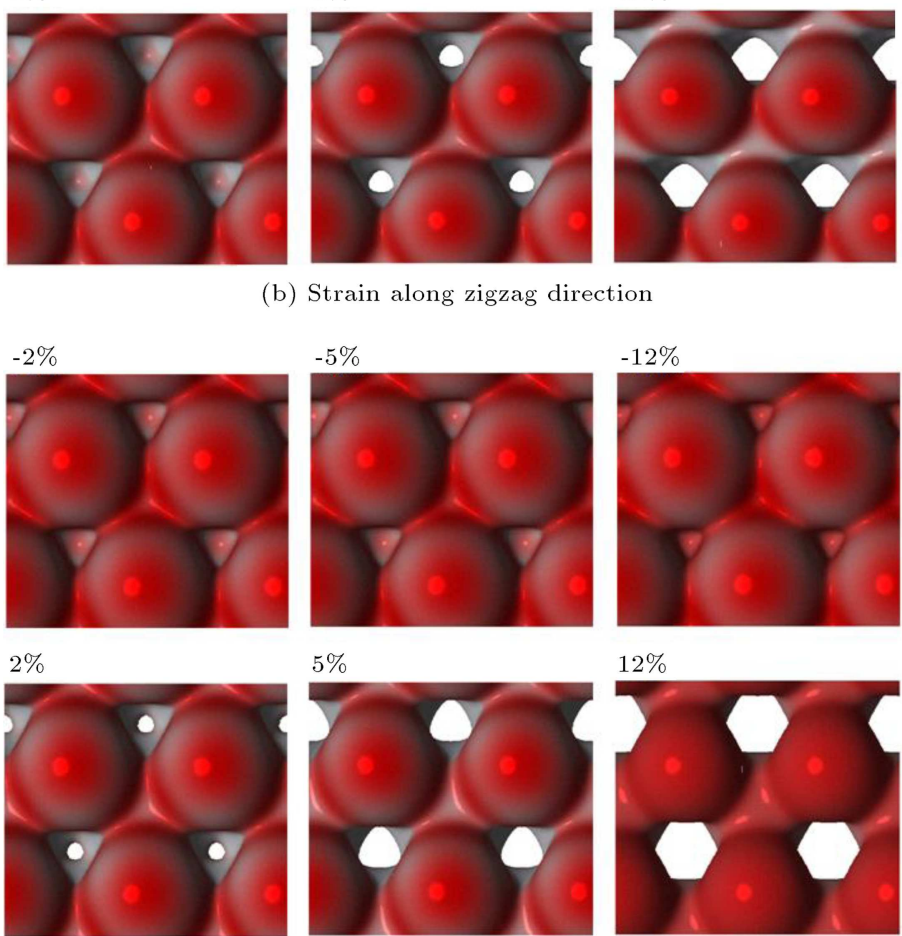

$12 \% \quad 0.08 \quad 0.20 \quad 3.47$

(c) Strain along biaxial direction

Figure 7. Top views of the electrostatic potential of $\mathrm{MoS}_{2}$ monolayer in the presence of the stain along the armchair direction, the zigzag direction, and the biaxial strain per 0.02 electrons/bohr ${ }^{3}$ isovalue electron density. The unit of the potential is Ry.

atom in down-layer. Thus, in order to obviously figure out the strain-induced potential due to three types of planar strain, the electrostatic potential maps of the strained $\mathrm{MoS}_{2}$ supercell are plotted in Figure 7(a)-(c) from top view. To determine the exact atomic position from potential point of view, the electrostatic potential maps of pristine $\mathrm{MoS}_{2}$ monolayer are presented per two different isovalues of 0.02 and 0.1 electrons/bohr ${ }^{3}$ in Figure 2(d)-(e), which correspond to the interstitial and atomic regions, respectively. To investigate the 
potential changes due to strain in all cases, the isosurface of the electron density with isovalue of 0.02 electrons $/$ bohr $^{3}$ is plotted. Figures show that the compressive strain causes the electrostatic potential of interstitial regions to drop in the $\mathrm{S}$ sheet. In other words, the electrostatic potential difference between interstitial and atomic regions decreases when the compressive strain increases. In contrast, the potential rise occurs in the interstitial regions in the $S$ sheet due to the tensile strain. These results confirm that the $\mathrm{S}$ atom takes more partial charge due to the compressive strain in comparison to the tensile one.

\section{Concluding remarks}

Electronic properties of the $\mathrm{MoS}_{2}$ monolayer under planar tensile and compressive strains were calculated using the DFT calculations. As a consequence of the strain, S atom took electron from Mo atom and became negatively charged. Besides these, the compressive strain induced larger partial charge changes than the tensile strain did. Also, strain could significantly cause the system to polarize. Moreover, the tensile and compressive strains induced the charge polarization in two opposite directions. Finally, the strain-induced charge polarization was proportional to the intensity value of strain.

\section{References}

1. Mak, K., Lee, C., Shan, J. and Heinz, T.F. "Atomically thin $\mathrm{MoS}_{2}$ : a new direct-gap semiconductor", Phys. Rev. Lett., 105, p. 136805-1 (2010).

2. Chang, J., Register, L.F. and Banerjee, S.K. "Atomistic full-band simulations of monolayer $\mathrm{MoS}_{2}$ transistors", Appl. Phys. Lett., 103, p. 223509 (4 pages) (2013).

3. Chhowalla, M., Shin, H.S., Eda, G., Li, L.-J., Loh, K.P. and Zhang, H. "The chemistry of two-dimensional layered transition metal dichalcogenide nanosheets", Nature Chem., 5, pp. 263-275 (2013).

4. Zeng, Z., Yin, Z., Huang, X., Li, H., He, Q., Lu, G., Boey, F. and Zhang, H. "Single-layer semiconducting nanosheets: high-yield preparation and device fabrication", Angew. Chem. Int. Ed. Engel., 50, pp. 11093-7 (2011).

5. Divigalpitiya, W.M.R., Morrison, S.R. and Frindt, R.F. "Thin oriented films of molybdenum disulphide", Thin Solid Films, 186, pp. 177-192 (1990).

6. Sahoo, M.P.K., Wang, J., Zhang, Y., Shimada, T. and Kitamura, T. "Modulation of gas adsorption and magnetic properties of monolayer- $\mathrm{MoS}_{2}$ by antisite defect and strain" J. Phys. Chem. C, 120, pp. 1411314121 (2016).

7. Shokri, A.A. and Salami, N. "Gas sensor based on $\mathrm{MoS}_{2}$ monolayer", Sens. Actuators B Chem., 236, pp. 378-385 (2016).
8. Radisavljevic, B., Radenovic, A., Brivio, J., Giacometti, V. and Kis, A. "Single-layer $\mathrm{MoS}_{2}$ transistors", Nature Nonetechnol., 6, pp. 147-150 (2011).

9. Salami, N., Shokri, A.A. and Elahi, S.M. "Tunable electronic and magnetic properties of a $\mathrm{MoS}_{2}$ monolayer with vacancies under elastic planar strain: $\mathrm{Ab}$ initio study", Physica E, 77, pp. 138-143 (2016).

10. Cheng, Y.C., Zhu, Z.Y., Mi, W.B., Guo, Z.B. and Schwingenschlögl, U. "Prediction of two-dimensional diluted magnetic semiconductors: Doped monolayer $\mathrm{MoS}_{2}$ systems", Phys. Rev. B, 87, pp. 100401-1100401-4 (2013).

11. Hua, A.-M., Wanga, L.-L., Mengc, B. and Xiao, W.-Z. "Ab initio study of magnetism in nonmagnetic metal substituted monolayer $\mathrm{MoS}_{2}$ ", Solid State Commun., 220, pp. 67-71 (2015).

12. Qi, J., Li, X., Chen, X. and Hu, K. "Strain tuning of magnetism in $\mathrm{Mn}$ doped $\mathrm{MoS}_{2}$ monolayer", J. Phys.: Condens. Matter, 26, pp. 256003-9 (2014).

13. Ghorbani-Asl, M., Borini, S., Kuc, A. and Heine, T. "Strain-dependent modulation of conductivity in single-layer transition-metal dichalcogenides", Phys. Rev. B, 87, pp. 235434(1)-235434(6) (2013).

14. Tabatabaei, S.M., Noei, M., Khaliji, K., Pourfath, M. and Fathipour, M. "A first-principles study on the effect of biaxial strain on the ultimate performance of monolayer $\mathrm{MoS}_{2}$-based double gate field effect transistor", J. Appl. Phys., 113, pp. 163708(1)-163708(6) (2013).

15. Johari, P. and Shenoy, V.B. "Tuning the electronic properties of semiconducting transition metal dichalcogenides by applying mechanical strains", ACS Nano, 6, pp. 5449-56 (2012).

16. Yun, W.S., Han, S.W., Hong, S.C., Kim, I.G. and Lee, J.D. "Thickness and strain effects on electronic structures of transition metal dichalcogenides: $2 \mathrm{H}-$ $\mathrm{MX}_{2}$ semiconductors $(M=\mathrm{Mo}, \mathrm{W} ; X=\mathrm{S}, \mathrm{Se}, \mathrm{Te})$ ", Phys. Rev. B, 85, pp. 033305(1)-033305(5) (2012).

17. Lu, P., Wu, X., Guo, W. and Zeng, X.C. "Straindependent electronic and magnetic properties of $\mathrm{MoS}_{2}$ monolayer, bilayer, nanoribbons and nanotubes", Phys. Chem. Chem. Phys., 14, pp. 13035-40 (2012).

18. Yu, S., Xiong, H.D., Eshun, K., Yuan, H. and Li, Q. "Phase transition, effective mass and carrier mobility of $\mathrm{MoS}_{2}$ monolayer under tensile strain", J. Appl. Surf. Sci., 325, pp. 27-32 (2015).

19. Rostami, H., Moghaddam, A.G. and Asgari, R. "Effective lattice Hamiltonian for monolayer $\mathrm{MoS}_{2}$ : Tailoring electronic structure with perpendicular electric and magnetic fields", Phys. Rev. B, 88, p. 085440 (2013).

20. Wu, W., Wang, L., Li, Y., Zhang, F., Lin, L., Niu, S., Chenet, D., Zhang, X., Hao, Y., Heinz, T.F., Hone, J. and Wang, Z.L. "Piezoelectricity of single-atomic-layer $\mathrm{MoS}_{2}$ for energy conversion and piezotronics", Nature, 514, pp. 470-474 (2014).

21. Duerloo, K.-A.N., Ong, M.T. and Reed, E.J. "Intrinsic piezoelectricity in two-dimensional materials", J. Phys. Chem. Lett., 3, pp. 2871-2876 (2012). 
22. Vanderbilt, D. and King-Smith, R.D. "Theory of polarization of crystalline solids", Phys. Rev. B, 48, pp. 4442-4455 (1993).

23. Bernardini, F., Fiorentini, V. and Vanderbilt, D. "Spontaneous polarization and piezoelectric constants of III-V nitrides", Phys. Rev. B, 56, pp. R10 024-027 (1997).

24. Vanderbilt, D. "Berry-phase theory of proper piezoelectricresponse", J. Phys. Chem. Solids, 61, pp. 147151 (2000).

25. Spaldin, N.A. "A beginner's guide to the modern theory of polarization", J. Solid State Chem., 195, pp. 2-10 (2012).

26. Li, W. and Li, J. "Piezoelectricity in two-dimensional group III monochalcogenides", Nano Res., 8, pp. 3796$3802(2015)$.

27. Ordejón, P., Artacho, E. and Soler, J.M. "Selfconsistent order-N density-functional calculations for very large systems", Phys. Rev. B, 53, pp. 10441-10444 (1996).

28. Soler, J.M., Artacho, E., Gale, J.D., Garca, A., Junquera, J., Ordejón, P. and Sánchez-Portal, D. "The SIESTA method for ab initio order-N materials simulation", J. Phys.: Condens. Matter, 14, pp. 27452779 (2002).

29. Troullier, N. and Martins, J.L. "Efficient pseudopotentials for plane-wave calculations", Phys. Rev. B, 43, pp. 1993-2006 (1991).
30. Ataca, C., Sahin, H., Aktürk, E. and Ciraci, S. "Mechanicaland electronic properties of $\mathrm{MoS}_{2}$ nanoribbons and their defects", J. Phys. Chem. C, 115, pp. 39343941 (2011).

31. Sawyer, C.B. and Tower, C.H. "Rochelle salt as a dielectric", Phys. Rev, 35, pp. 269-273 (1930).

\section{Biographies}

Nadia Salami received MSc degree in Condensed Matter Physics in 2006 and $\mathrm{PhD}$ in exact results on gas sensor based on $\mathrm{MoS}_{2}$ nanostructures, in January 2016, from Science and Research Branch, Islamic Azad University in Tehran, Iran. Her supervisor was Professor A. A. Shokri. Now, she is an Assistant Professor in the Department of Physics, Islamic Azad University, Yasooj, Iran.

Aliasghar Shokri obtained an MSc degree in Condensed Matter Physics in 2000 and a PhD in Spin Electronics Transport in Magnetic Multilayers and Nanostructures in February 2005 from Sharif University of Technology (SUT) in Tehran, Iran. His supervisor was Professor K. Esfarjani. Now, he is a Professor in the Department of Physics, Tehran Payame Noor University (PNU), and a Resident Researcher on theoretical Nano-Science in the Department of NanoScience, Institute for Studies in Theoretical Physics and Mathematics (IPM), Tehran, Iran. 